\title{
Mesoporous silicon sponge as an anti-pulverization structure for high-performance lithium-ion battery anodes
}

Xiaolin $\mathrm{Li}^{1}$, Meng $\mathrm{Gu}^{2}$, Shenyang $\mathrm{Hu}^{1}$, Rhiannon Kennard ${ }^{3}$, Pengfei Yan ${ }^{2}$, Xilin Chen ${ }^{1}$, Chongmin Wang ${ }^{2}$, Michael J. Sailor ${ }^{3}$, Ji-Guang Zhang ${ }^{1} \&$ Jun Liu $^{1}$

Nanostructured silicon is a promising anode material for high-performance lithium-ion batteries, yet scalable synthesis of such materials, and retaining good cycling stability in high loading electrode remain significant challenges. Here we combine in-situ transmission electron microscopy and continuum media mechanical calculations to demonstrate that large ( $>20 \mu \mathrm{m}$ ) mesoporous silicon sponge prepared by the anodization method can limit the particle volume expansion at full lithiation to $\sim 30 \%$ and prevent pulverization in bulk silicon particles. The mesoporous silicon sponge can deliver a capacity of up to $\sim 750 \mathrm{mAhg}^{-1}$ based on the total electrode weight with $>80 \%$ capacity retention over 1,000 cycles. The first cycle irreversible capacity loss of pre-lithiated electrode is $<5 \%$. Bulk electrodes with an area-specific-capacity of $\sim 1.5 \mathrm{mAh} \mathrm{cm}^{-2}$ and $\sim 92 \%$ capacity retention over 300 cycles are also demonstrated. The insight obtained from this work also provides guidance for the design of other materials that may experience large volume variation during operations.

\footnotetext{
${ }^{1}$ Energy and Environment Directorate, Pacific Northwest National Laboratory, Richland, Washington 99352, USA. ${ }^{2}$ Environmental and Molecular Sciences Laboratory, Pacific Northwest National Laboratory, Richland, Washington 99352, USA. ${ }^{3}$ Department of Chemistry and Biochemistry, University of California, San Diego, La Jolla, California 92093, USA. Correspondence and requests for materials should be addressed to J.-G.Z. (email: jiguang.zhang@pnnl.gov) or to J.L. (email: jun.liu@pnnl.gov).
} 
ncreasing demands for high energy density, long cycle life, and low-cost lithium (Li)-ion batteries in critical applications such as electrical vehicles and portable devices have stimulated extensive research interest in developing novel electrode materi$\mathrm{als}^{1,2}$. Among all the candidates for anode materials, silicon (Si) has a theoretical specific capacity of $\sim 4,200 \mathrm{mAhg}^{-1}\left(\mathrm{Li}_{22} \mathrm{Si}_{5}\right)$, $\sim 11$ times of the theoretical specific capacity of the state-of-theart graphite anode, and it does not have the safety concern of lithium metal dendrite formation ${ }^{3}$. Therefore, Si has been regarded as one of the most promising anode materials for next-generation Li-ion batteries. However, its large volume expansion $(>300 \%)$ during the lithiation process leads to the pulverization of silicon particles $(>200 \mathrm{~nm})$ and subsequently results in fast capacity fade of the electrode ${ }^{3}$. Many in-situ studies have directly observed the intrinsic volume expansion and pulverization of $\mathrm{Si}$ particles ${ }^{4-6}$. To overcome/bypass the pulverization problem, tremendous effort has been made on the synthesis of ultra-fine Si nanoparticles $(<50 \mathrm{~nm})$, development of new binders, and design of novel nanostructured Si materials, such as nanowires, nanotubes, hollow spheres and core-shell structures ${ }^{7-32}$. Milestone performance with a capacity of $\sim 600 \mathrm{mAh} \mathrm{g}^{-1}$ and $\sim 88 \%$ capacity retention over 6,000 cycles at a $12 \mathrm{C}$ rate has been demonstrated with double-walled $\mathrm{Si}$ nanotubes at a low mass loading ${ }^{11}$. Recently, over 100 stable cycles has also been demonstrated with a high loading $\mathrm{Si}$ anodes using pomegranate-like Si-C yolk-shell structures ${ }^{33}$. However, scalable synthesis of nanostructured Si materials that retain good long-term cycling stability in bulk electrodes of high mass loading remains a significant challenge. In general, the cycling stability of nanostructured $\mathrm{Si}$ anodes degrades rapidly as the mass loading or the electrode thickness increases to $>10 \mu \mathrm{m}$. Methods are needed to prepare bulk Si materials without structural degradation during lithiation/de-lithiation. Recently, porous structured $\mathrm{Si}$ and $\mathrm{Si}$ based composites have attracted significant attention ${ }^{14,24-32}$. The idea is to pre-form meso/macropores in the $\mathrm{Si}$ structure to accommodate the large volume expansion that accompanies the lithiation process. Newly developed synthetic chemistries and approaches that lead to significantly improved performance have been demonstrated. However, the suppression of pulverization of bulk Si particles, and long-term cycling stability at high Si loading conditions have not been demonstrated. The existing work on $\mathrm{Si}$ anodes has not yet met the requirements for commercial applications because of unsatisfactory performance or serious challenges associated with cost and scale-up.

In this study, we synthesize large $(>20 \mu \mathrm{m})$ mesoporous $\mathrm{Si}$ sponge (MSS) using an electrochemical etching method and demonstrated its excellent performance as anodes for Li-ion batteries. The MSS has a highly porous structure with thin crystalline $\mathrm{Si}$ walls surrounding large pores that are up to $\sim 50 \mathrm{~nm}$ in diameter. In-situ transmission electron microscopy (TEM) shows that the volume change of the Si walls during the charge/discharge processes is primarily accommodated by the inner pores in the MSS, leading to only $\sim 30 \%$ expansion in the full particle size instead of $>300 \%$ expansion as in the case of conventional Si particles, and the particles do not pulverize even after one thousand charge/discharge cycles. With this unique structure, a MSS anode delivers a capacity of up to $\sim 750 \mathrm{mAh} \mathrm{g}^{-1}$ based on the whole electrode weight including binder and conductive carbon, and retains more than $80 \%$ capacity over 1,000 cycles. Its first cycle irreversible capacity loss can be reduced to $<5 \%$ by pre-lithiation with $\mathrm{Li}$ powder. The MSS-based anode also demonstrates $\sim 92 \%$ capacity retention over 300 cycles at a high area-specific-capacity of $\sim 1.5 \mathrm{mAh} \mathrm{cm}^{-2}$. Furthermore, the thick electrodes with an areal capacity of $\sim 3$ and $4 \mathrm{mAh} \mathrm{cm}^{-2}$ also demonstrate a capacity retention of $>96 \%$ over 70 cycles. These results suggest that stable bulk $\mathrm{Si}$ anode materials are possible even at deep lithiation and high loading conditions required for practical applications.

\section{Results}

Calculation of the MSS volume expansion. The large volume expansion of Si during lithiation is intrinsic; however, the effects of the expansion such as the pulverization of bulk/micron-sized $\mathrm{Si}$ particles can be alleviated or eliminated by rational design of the Si structure. With the help of theoretical calculations, we identified a MSS structure with inner pores that can accommodate most of the volume change so that the overall particle experiences minimal expansion. Figure 1a schematically illustrates the structural change of a model of cylindrical MSS particle with cylindrical pores during the lithiation/de-lithiation processes. The solid $\mathrm{Si}$ portion of the particle is predicted to experience a $\sim 300 \%$ volume increase at the full lithiation state. However, the dimension $(a, h)$ of the particle experiences only a small increase/ expansion during lithiation if the void/pore radius $(r)$ decreases sufficiently. Based on the assumption of stress-free or volume conservation, the effect of the initial pore radius $\left(r_{1}\right)$ and the porosity/void volume fraction $\left(V_{f}\right)$ on the average volume change can be estimated and optimized. For a MSS particle $\left(a_{1}, h_{1}\right)$ with given porosity $\left(V_{f}=80 \%\right), \mathrm{Si}$ wall thickness $(\sim 10 \mathrm{~nm})$, initial pore radius $\left(r_{1}=10 \mathrm{~nm}\right)$, the expansion rates of the cylindrical particle along the radial direction $(\kappa)$, along the axial direction $(\xi)$ and the pore radius after lithiation $\left(r_{2}\right)$ are calculated and plotted in Fig. 1b. The pore volume after full lithiation is predicted to be $75 \%$ less and the radius $\left(r_{2}\right)$ will be approximately half of the initial pore radius assuming the overall particle does not expand at all (in real cases, the ratio of the expansion towards the inner pores and outside dimensions depends on the Li-ion diffusion and other factors such as the structure confinement effect). Similarly, we estimated the upper and lower bounds of particle

a
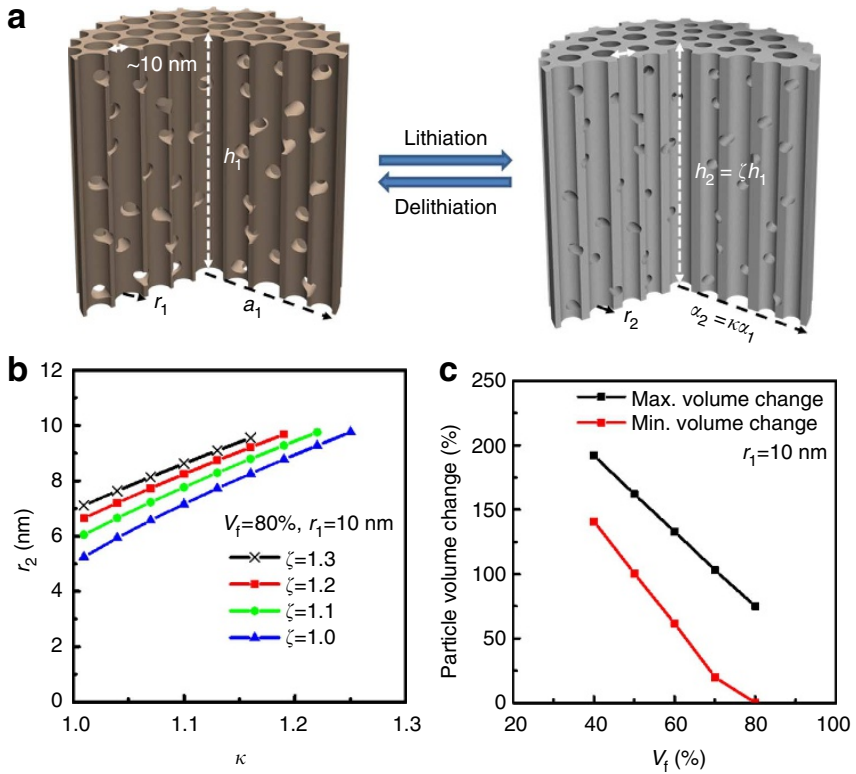

Figure 1 | The MSS model structure and theoretical estimation of its structure change after lithiation. (a) A schematic model of the MSS particle. (b) The correlation of the pore radius after full lithiation $\left(r_{2}\right)$, and the expansion rates of the particle along the radical direction $(\kappa)$ and along the axial direction ( $\xi) . r_{1}=10 \mathrm{~nm} ; V_{f}=80 \%$. The silicon wall thickness is $10 \mathrm{~nm}$. (c) The plot of the MSS particle volume expansion versus porosity $\left(V_{f}\right) . r_{1}=10 \mathrm{~nm}$. The Si wall thickness is $10 \mathrm{~nm}$. 
volume changes as a function of $V_{f}$ and initial pore radius $\left(r_{1}\right)$ under the following constraint conditions: $r_{2} \geq 0, \kappa \geq 1$ and $\xi \geq 1$. Figure $1 \mathrm{c}$ shows that the overall volume expansion decreases almost linearly with the increase of void volume fraction $\left(V_{f}\right)$ in the MSS particle. The volume change decreases to between 0 and $75 \%$ if the MSS particle has $\sim 80 \%$ porosity $\left(V_{f}=80 \%\right)$, thin $\mathrm{Si}$ walls of $\sim 10 \mathrm{~nm}$ and large initial pore radius $\left(r_{1}=10 \mathrm{~nm}\right)$. Systematic calculations on the influence of porosity $\left(V_{f}\right)$ and initial pore radius $\left(r_{1}\right)$ on the particle volume expansion were performed; the results are given in the Supplementary Figs 1 and 2.

Preparation and characterization of the desired MSS structure. The preparation of porous Si by anodic electrochemical etching of crystalline Si has been known for more than 50 years $^{34}$. The process allows the designed fabrication of various pore sizes (between $2 \mathrm{~nm}$ to several $\mu \mathrm{m}$ ) and porosities (ranging from 5-95\%) and it has been exploited for applications such as bioimaging reagents and controlled drug delivery $35-37$. Here we tailored the etching protocol to synthesize MSS with a high porosity suitable for rechargeable Li-ion battery applications (See Methods). Figure 2a,b shows typical scanning electron microscope (SEM) images of the MSS. At low magnification (Fig. 2a), the MSS particles have the appearance of irregular bricks or rocks with overall dimensions of $>\sim 20 \mu \mathrm{m}$. The highly porous sponge-like structure of the particles is revealed at higher magnifications (Fig. 2b), in which large pores with diameters up to $\sim 50 \mathrm{~nm}$ are surrounded by thin $(\sim 10 \mathrm{~nm}) \mathrm{Si}$ walls. Figure $2 \mathrm{c}$ shows the TEM image of a thin slide from a bulk MSS particle. The pores are up to $50 \mathrm{~nm}$ in diameter surrounding with thin walls of $\sim 8 \mathrm{~nm}$, in consistent with the SEM results. High-solution TEM images (Supplementary Fig. 3) show that the MSS wall consists of a crystalline Si skeleton coated with a thin amorphous silicon layer of $\sim 1-3 \mathrm{~nm}$ in thickness. The thin amorphous layer is silicon oxide formed during exposure of the material to air. The energydispersive $\mathrm{x}$-ray spectroscopy analysis shows that the MSS has
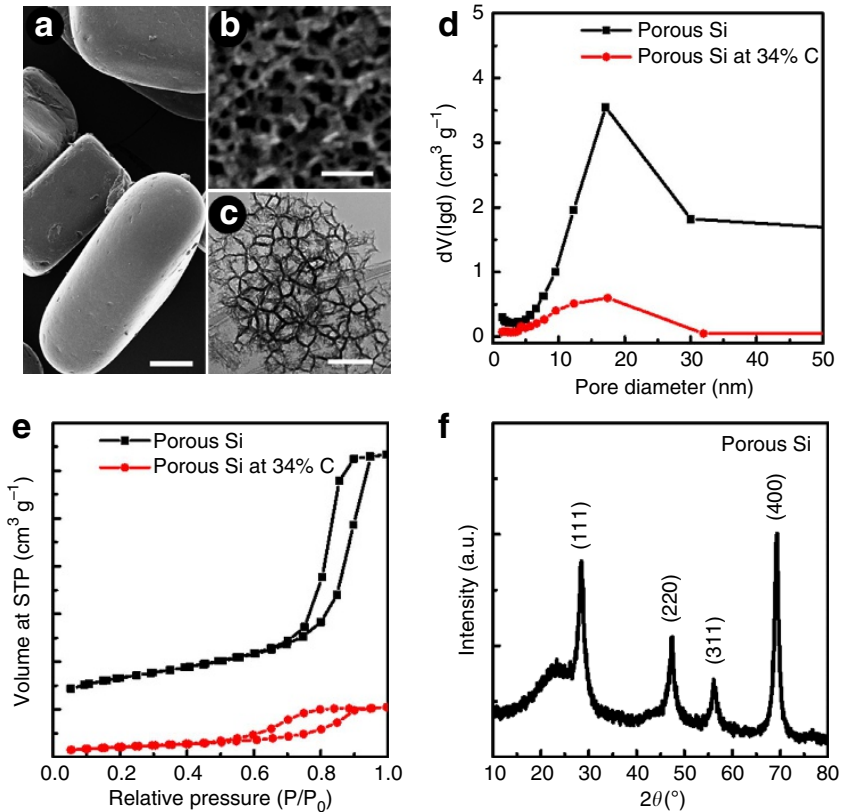

Figure 2 | Structure of the MSS. ( $\mathbf{a}$ and $\mathbf{b}$ ) SEM images of the MSS at different magnifications. (c) TEM image of the MSS. The scale bar in $\mathbf{a}, \mathbf{b}$ and $\mathbf{c}$ is $20 \mu \mathrm{m}, 100 \mathrm{~nm}$ and $100 \mathrm{~nm}$, respectively. (d) and (e) Pore size distribution and isothermal curves of the MSS sample before and after carbon deposition. (f) XRD pattern of the MSS material. $\sim 19$ wt\% oxygen content (see Supplementary Method 1). This oxide layer may lower the capacity and induce first cycle irreversible capacity loss (vide infra).

Nitrogen gas sorption measurements (Fig. 2d,e) were conducted to investigate the pore size distribution, surface area and porosity of the MSS. The pore diameter of the MSS is $\sim 17.1 \mathrm{~nm}$ (Fig. 2d, calculated from the adsorption branch using the BarrettJoyner-Halenda method; see detailed results in Supplementary Table 1.) and the surface area is $\sim 494.7 \mathrm{~m}^{2} \mathrm{~g}^{-1}$ (calculated using the Brunauer-Emmett-Teller theory). The pore volume is $\sim 1.7 \mathrm{~cm}^{3} \mathrm{~g}^{-1}$ (Supplementary Table 1) and hence the porosity is $\sim 80 \%$. This MSS closely approximates the calculated optimal structure. The x-ray diffraction (XRD) pattern (Fig. 2f) shows that the MSS consists of crystalline $\mathrm{Si}$ and amorphous oxide, in consistent with the TEM results (Fig. $2 c$ and Supplementary Fig. 3).

To improve the electrical conductivity, the as-prepared MSS material was coated with amorphous carbon using chemical vapour deposition (see Methods) ${ }^{38}$. The Brunauer-Emmett-Teller surface area $\left(155.8 \mathrm{~m}^{2} \mathrm{~g}^{-1}\right)$ and pore volume $\left(0.33 \mathrm{~cm}^{3} \mathrm{~g}^{-1}\right)$ of the MSS significantly decreases (Fig. 2d,e and Supplementary Table 1) after carbon coating, indicating that the carbon is deposited both inside the pores and on the surface of the MSS particles. Thermogravimetric analysis revealed a carbon content of $\sim 34$ wt\% (Supplementary Fig. 4). We believe that the carbon coating not only improves the conductivity of the MSS, but also mechanically/electrically integrates the particle during cycling.

In-situ TEM experiment. The volume expansion of the MSS particle under cycling conditions was quantitated by in-situ TEM during battery operation (see Methods). The in-situ TEM battery experiment is schematically shown in Fig. 3a. A carbon-coated MSS particle is loaded onto a Si nanowire, which is then connected to the $\mathrm{Li}_{2} \mathrm{O} / \mathrm{Li}$ end. The $\mathrm{Si}$ nanowire lithiates first and transports $\mathrm{Li}$ to the MSS particle. As shown in Fig. 3b, the dimension of the pristine MSS particle is characterized by the length in two directions: parallel and perpendicular to the pore direction. The small red arrow in the Figure indicates the location on the particle where the measurement was performed. The dimension and crystallinity of MSS at different stages of lithiation were monitored by TEM (Fig. 3b-e). Before lithiation, the pristine particle was $1,219 \mathrm{~nm}$ in length (perpendicular to the pore direction) and $488 \mathrm{~nm}$ in width (parallel to the pore direction) (Fig. 3b). The corresponding selected area electron diffraction pattern indicates that the direction perpendicular to the pore direction is the $\langle 01-1\rangle$ direction, which is consistent with the $<100>$ orientation of pores typically observed with this type of porous $\mathrm{Si}^{35}$. As lithiation continued to $22 \mathrm{~min}$ (Fig. 3c), the length increased to $1295 \mathrm{~nm}$ while the width increased to $508 \mathrm{~nm}$. The diffraction pattern at $22 \mathrm{~min}$ showed faint diffraction spots, indication of the lowering of crystallinity induced by the lithiation process. After $40 \mathrm{~min}$ of lithiation, the particle showed a completely amorphous structure as exhibited by the ring patterns of the diffraction pattern in Fig. 3d. At the same time, the length increased to $1,315 \mathrm{~nm}$ and the width increased to $530 \mathrm{~nm}$. After an extended period of lithiation $(160 \mathrm{~min})$, the sample was fully lithiated. At this point the sample showed no further increase in length, and the width increased to $552 \mathrm{~nm}$. The diffraction pattern at this final lithiation state indicated that the structure was amorphous, and the electron energy loss spectrum (Supplementary Fig. 5) indicated a Li:Si atomic ratio of 3.75, which corresponds to full lithiation at room temperature ${ }^{3}$.

Electrochemical performance. To further corroborate the stability of the MSS structure against pulverization during lithium 
a

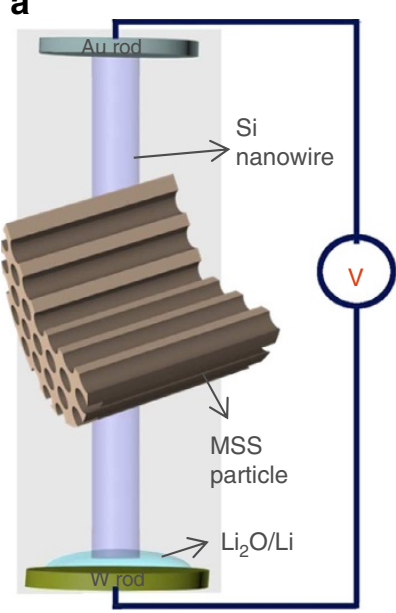

b

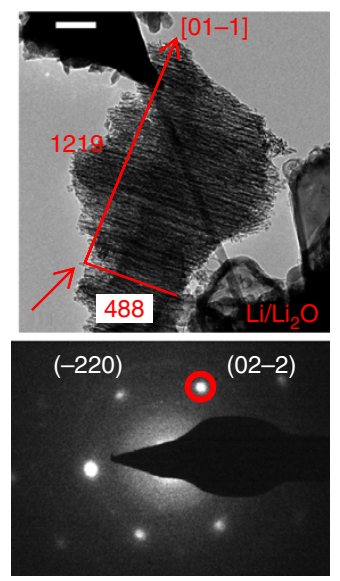

C

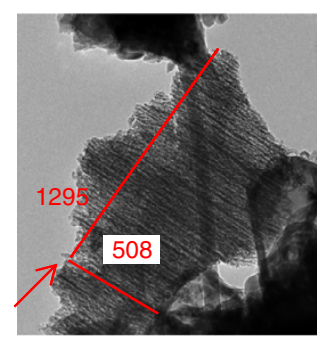

d

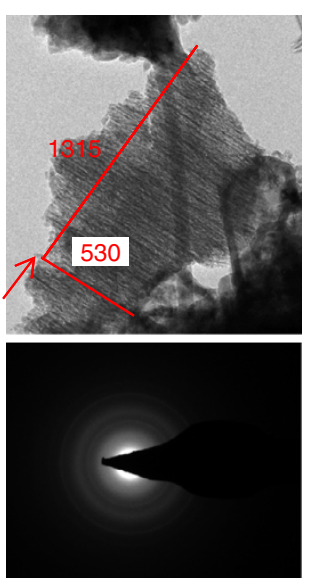

e

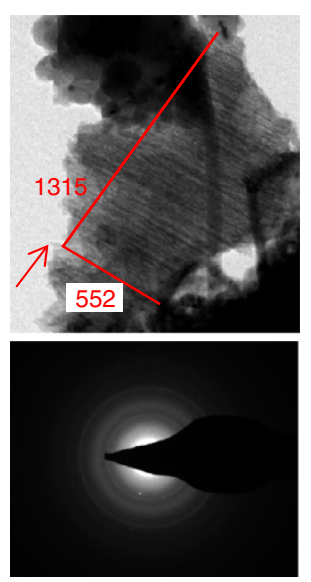

Figure 3 | In-situ TEM observation of the lithiation process of an MSS particle. (a) Schematic figure of the in-situ TEM battery experiment. (b-e) TEM images and selected area electron diffraction patterns of the MSS particle at different lithiation states: (b) before lithiation; (c) 22 min of lithiation; (d) $40 \mathrm{~min}$ of lithiation; (e) $160 \mathrm{~min}$ of lithiation. Scale bar, $200 \mathrm{~nm}$ (b-e).

insertion/extraction processes, MSS particles have been used to prepare anode and tested in lithium batteries (see Methods). Unlike conventional micron-sized Si particles that exhibit fast capacity fading ${ }^{3}$, the micron-sized MSS demonstrated superior electrochemical performance. Figure $4 \mathrm{a}$ shows the cyclic voltammetry curve of an anode prepared from the MSS material. The curves appear very similar to those obtained from standard crystalline $\mathrm{Si}^{3}, 16$, and they are consistent with the crystalline XRD pattern shown in Fig. $2 \mathrm{~d}$. The cathodic peak at $\sim 0.21 \mathrm{~V}$ and the anodic peaks at $\sim 0.32$ and $\sim 0.49 \mathrm{~V}$ are characteristic of amorphous $\mathrm{Si}$, and the cathodic peak at $\sim 0 \mathrm{~V}$ is characteristic of both crystalline and amorphous $\mathrm{Si}^{16}$. In the first cathodic scan, only crystalline Si exists, so only one peak at $\sim 0 \mathrm{~V}$ is observed. However, the crystalline structure becomes amorphous during lithiation, and hence, two anodic peaks of amorphous $\mathrm{Si}$ (at $\sim 0.32$ and $\sim 0.49 \mathrm{~V}$ ) are observed in the subsequent anodic scans.

The charge-discharge curves (Fig. $4 \mathrm{~b}$ ) obtained between 0.05 and $1.5 \mathrm{~V}$ are similar to those observed with other nano-Si materials. A low Coulombic efficiency and relatively large irreversible capacity loss occurred during the first cycle, which is related to formation of the solid-electrolyte interphase and the high surface oxygen content in the surface of MSS and consistent with the amorphous signal appearing at $2 \theta=24^{\circ}$ in the XRD pattern (Fig. 2f). The Coulombic efficiency for the electrodes without pre-lithiation treatment is $\sim 56 \%$ in the 1 st cycle and then it quickly increased to $\sim 99.7 \%$. The carboncoated MSS displayed very good long-term cycling stability and a high capacity (up to $\sim 750 \mathrm{mAh} \mathrm{g}^{-1}$ ). The specific capacity values reported in this work are based on the weight of the full electrode, including the MSS material, binder and carbon additive (see Methods). Figure $4 \mathrm{c}$ shows the representative results of an electrode with $\sim 46$ wt $\%$ MSS. It has a specific capacity of $\sim 640 \mathrm{mAh}^{-1}$ and $\sim 81 \%$ capacity retention over 1,000 cycles. By tuning the weight ratio of the MSS in electrodes, we can fine tune the capacity and the retention for different applications. Figure $4 \mathrm{~d}$ shows the long-term cycling data for a battery with $\sim 40 \mathrm{wt} \%$ MSS in the electrode. A capacity of $\sim 570 \mathrm{mAh} \mathrm{g}^{-1}$ and $\sim 87 \%$ capacity retention over 1,000 cycles were obtained (Fig. $4 \mathrm{~d}$ ). The capacity ramping-up process in the first 100 cycles can be attributed to the fact that the MSS has a large particle size; hence, it takes many cycles to fully utilize its full capacity.
The large first cycle irreversible capacity loss, which would be a deficiency for practical applications, can be solved by pre-lithiation of the MSS anode (See detail pre-lithiation procedure and charge/ discharge profile of pre-lithiated MSS in Supplementary Fig. 6 in the Supplementary Materials). By adding an appropriate amount of stabilized Li metal powder (SLMP, FMC Lithium) to the MSS electrodes, the irreversible capacity loss in the first cycle was greatly reduced. As shown in Fig. 4e,f, pre-lithiated MSS electrodes displayed first cycle capacity losses as low as $\sim 5 \%$ (Fig. 4e) and $\sim 1 \%$ (Fig. 4f). The Coulombic efficiency for the electrodes with pre-lithiation treatment is $\sim 94.5 \%$ for the 1st cycle and then it increased to $>99.4 \%$. As indicated in Fig. 4e,f, high capacities of $\sim 740 \mathrm{mAh}^{-1}$ and $\sim 650 \mathrm{mAhg}^{-1}$ and $\sim 80 \%$ capacity retention over 800 cycles were obtained. In spite of its large particle size, MSS particles have demonstrated very good rate performance. Figure $4 \mathrm{f}$ shows that the capacity is $\sim 790 \mathrm{mAhg}^{-1}$ at $0.1 \mathrm{Ag}^{-1}$ current density, $\sim 630 \mathrm{mAhg}^{-1}$ at $1 \mathrm{Ag}^{-1} \sim 530 \mathrm{mAhg}^{-1}$ at $2 \mathrm{Ag}^{-1}$, and $\sim 410 \mathrm{mAhg}^{-1}$ at $4 \mathrm{Ag}^{-1}$. The capacity retention is $\sim 82 \%$ after 800 cycles ( 10 cycles each at current densities of 0.1 , $0.25,0.5,1,2$, and $4 \mathrm{Ag}^{-1}$ and then 740 cycles at $\left.1 \mathrm{Ag}^{-1}\right)$, which demonstrates the ability of the MSS to recover after being subjected to high charge/discharge rates.

Post-analysis of the electrode by SEM confirms the excellent structural stability of the MSS. As shown in Supplementary Fig. 7, MSS particles before and after 1,000 cycles exhibit similar size $(>20 \mu \mathrm{m}) \quad$ (Supplementary Fig. 7a,b) and size distribution (Supplementary Fig. 7c). The particles are coated with a layer of the solid-electrolyte interphase but they are not broken (Supplementary Fig. 7b). To further examine if cracks exist inside the large particles, we checked the cross-section of randomly picked large MSS particles after cycling. Supplementary Fig. 8 shows that the MSS particle still has similar porous structure and there is no cracking in the MSS after 1,000 cycles.

With the pulverization eliminated and particle volume expansion limited to $\sim 30 \%$, the MSS material allows good cycling stability for electrodes with high $\mathrm{Si}$ loading per unit area. A high area-specific-capacity of $\sim 1.50 \mathrm{mAh} \mathrm{cm}^{-2}$ at $0.5 \mathrm{~mA} \mathrm{~cm}^{-2}$ discharge and $0.75 \mathrm{~mA} \mathrm{~cm}^{-2}$ charge current density has been demonstrated (Fig. 5a). It shows excellent cycling stability with $\sim 92 \%$ capacity retention over 300 cycles. The corresponding specific capacity is $\sim 750$ and $950 \mathrm{mAh} \mathrm{g}^{-1}$ at low current density of $0.06 \mathrm{~mA} \mathrm{~cm}^{-2}$ (Supplementary Fig. 9). The pre-lithiated high loading MSS electrodes have very similar 

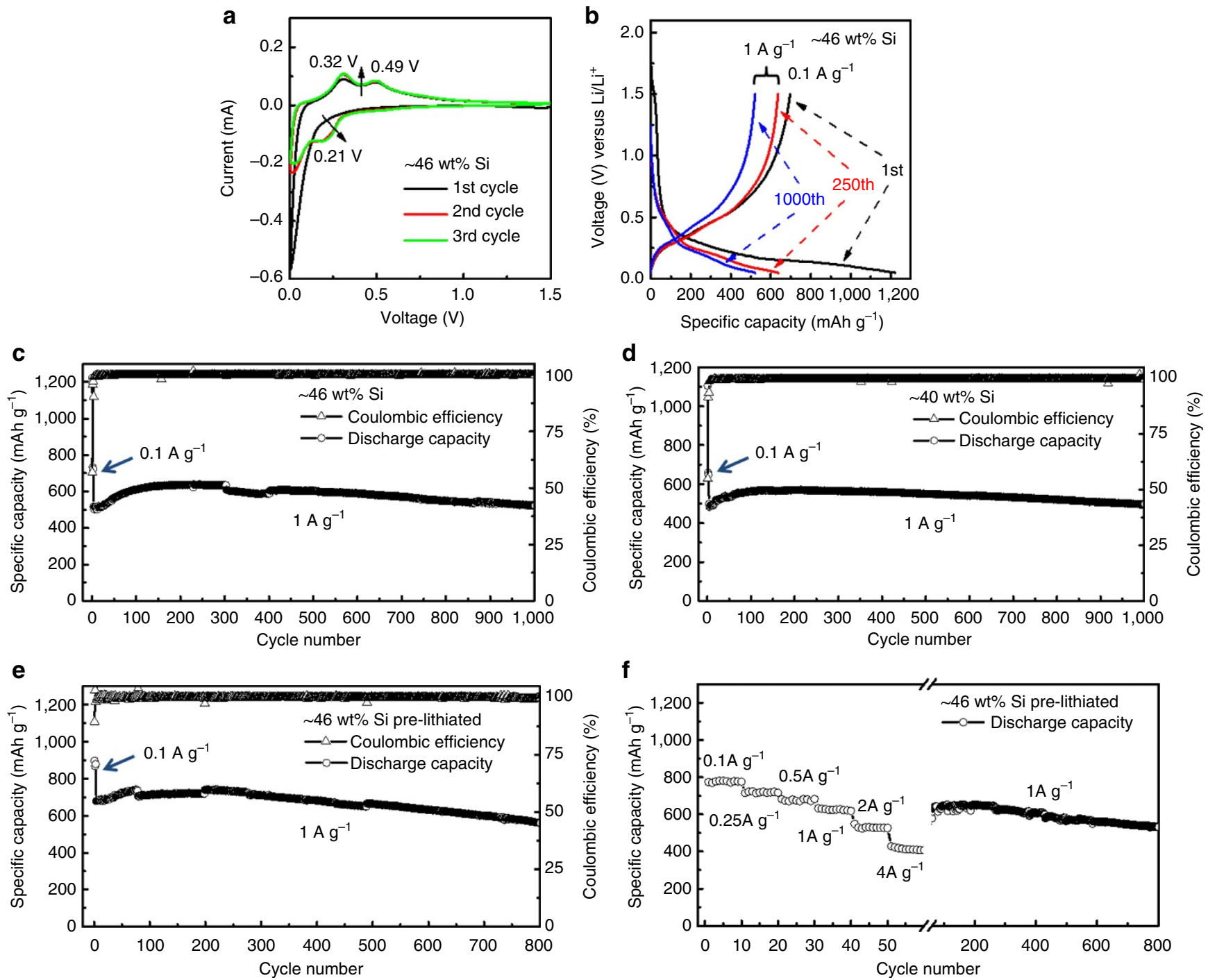

Figure 4 | Electrochemical performance of the MSS-based anodes. (a) Cyclic voltammogram curves of a typical MSS anode. (b) Typical charge-discharge profiles of the MSS anode with 46 wt\% MSS loading. (c) Long-term cycling of the MSS electrode in $\mathbf{b}$. (d) Long-term cycling of an electrode with $\sim 40 \mathrm{wt} \%$ MSS loading. (e) Long-term cycling of a pre-lithiated electrode with $\sim 46 \mathrm{wt} \%$ MSS loading. (f) Rate performance of a pre-lithiated electrode with $\sim 46$ wt $\%$ MSS loading.

initial capacity while the cycling stability may be slightly different as a result of the hand-pressing pre-lithiation treatment procedure. Supplementary Fig. 10 shows another pre-lithiated electrode with an areal capacity of $\sim 1.44 \mathrm{mAh} \mathrm{cm}^{-2}$ and $\sim 90.7 \%$ capacity retention over 300 cycles. These thick electrodes without (Supplementary Fig. 10a) and with pre-lithiation treatment (Supplementary Fig. 10b) can have excellent extended cycling performance with $\sim 85 \%$ capacity retention over 450 cycles.

High loading electrodes with the areal capacity up to $\sim 3$ and $4 \mathrm{mAh} \mathrm{cm}^{-2}$ were also demonstrated. Supplementary Figure 11a shows the cycling performance of three cells with loading of $\sim 3.5-4 \mathrm{mg} \mathrm{cm}^{-2}$. The areal capacity is $\sim 4.0 \mathrm{mAh} \mathrm{cm}^{-2}$ (3.7, 3.9 and $4.2 \mathrm{mAh} \mathrm{cm}^{-2}$, respectively) at a charge/discharge current density of $0.06 \mathrm{mAh} \mathrm{cm}^{-2}$ and $\sim 3 \mathrm{mAh} \mathrm{cm}^{-2}(2.6,2.8$ and $3.1 \mathrm{mAh} \mathrm{cm}^{-2}$, respectively) at $0.5 \mathrm{~mA} \mathrm{~cm}^{-2}$ discharge and $0.75 \mathrm{~mA} \mathrm{~cm}^{-2}$ charge current density. The capacity retention is $>96 \%$ after 70 cycles. With the loading further increased to $\sim 5.3 \mathrm{mg} \mathrm{cm}^{-2}$, the areal capacity can be $>4 \mathrm{mAh} \mathrm{cm}^{-2}$ at $0.5 \mathrm{~mA} \mathrm{~cm}^{-2}$ discharge and $0.75 \mathrm{~mA} \mathrm{~cm}^{-2}$ charge current density (Supplementary Fig. 11b).

\section{Discussion}

We postulate that the suppression of pulverization and the low volume expansion of MSS particles are the result of three factors: (1) the pores in the MSS particle serve as buffers that accommodate the volume expansion of $\mathrm{Si}$ during lithiation; (2) the $\sim 10 \mathrm{~nm} \mathrm{Si}$ walls are sufficiently thin that they reversibly expand/shrink during lithiation/de-lithiation without breaking ${ }^{7}$; and (3) the solid surface oxide layer formed at the pore wall surface serves to confine and reinforce the nanostructure ${ }^{11}$. We believe that these three factors together result in the unique structure of the MSS that leads to its desirable properties. This reasoning also explains the observation that the volume change revealed by the in-situ TEM study is somewhat smaller than those predicted by the theoretical calculations which does not count the effect of solid surface oxide layer.

The calculations indicate that an optimal MSS particle should have a high porosity $\left(V_{f}>70 \%\right)$, thin Si walls (thickness $<\sim 10$ $\mathrm{nm})$ and large pore radius $\left(r_{1}>\sim 10 \mathrm{~nm}\right)$ to preserve a relatively small overall volume variation $(<75 \%)$ during lithiation/delithiation processes. It must be noted that the maximum volume expansion of the MSS particle only decreases $17 \%$ (from 75 to 

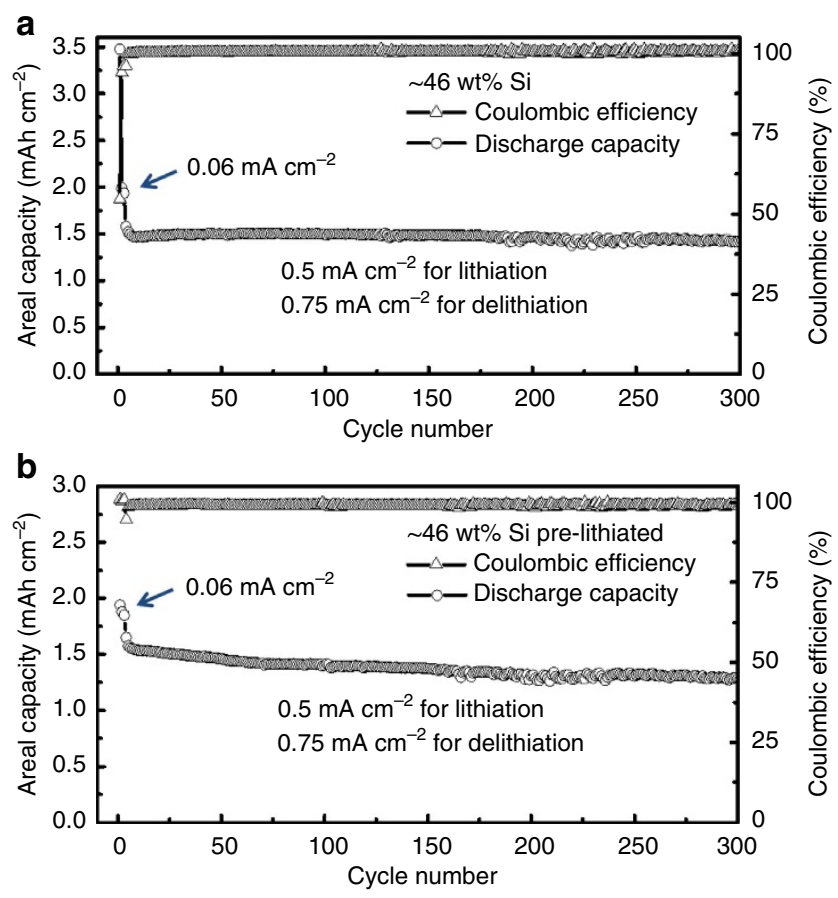

Figure 5 | Cycling stability of the MSS-based anodes with high areal discharge capacity of $\sim \mathbf{1 . 5} \mathrm{mAh} \mathrm{cm}^{-2}$. (a) A typical MSS anode. (b) A pre-lithiated MSS anode.

$58 \%$ ) when the pore radius increases $150 \%$ from 10 to $25 \mathrm{~nm}$. This suggests that $\sim 10 \mathrm{~nm}$ pore radius can effectively accommodate the volume expansion. Further increase in pore size could lead to a decrease in the volumetric capacity of the electrode.

It is well-known that $\mathrm{Si}$ shows significant anisotropic expansion upon lithiation, with an elongation of $\sim 200 \%$ along the family of $<011>$ directions ${ }^{39,40}$. Si particles with sizes $>200 \mathrm{~nm}$ will pulverize during the lithiation process ${ }^{4,5}$. In the case of MSS, the channel of the pores in the porous $\mathrm{Si}$ is along $<100>$ direction. Therefore, the expected large expansion of $\mathrm{Si}$ is along pore radius direction and can be absorbed by the open pores. This will effectively minimize the effect of volume expansion caused by lithiation process. In fact, the observed expansion for the whole particle along the $\langle 011\rangle$ direction perpendicular to the pore channels is only $\sim 7.8 \%$. The small expansion observed along the pore direction (that is, $\langle 100\rangle$ direction) is also consistent with the previous reports 39,40 . As a result, the MSS studied in this work exhibits the preferred pore orientation and pore structure which can effectively absorb the volume expansion inside of its nanostructure and prevent the particle from pulverization.

For purpose of discussion we describe the three-dimensional MSS particle in terms of $x, y$, and $z$ directions, where the $x$ direction $(<100>)$ is parallel to the pore direction and the $y(<01-1>)$ and the $z(<011>)$ directions are both perpendicular to the pore direction ( $y$ and $z$ are in the same direction family and have identical volume expansion.). The total volume expansion of the particle can be approximately written as $x_{f}^{*} y_{f}^{*} z_{f}-x_{0}^{*} y_{0}^{*} z_{0}$ (where $x_{f}, y_{f}, z_{f}$ are the coordinates after full lithiation, while $x_{0}, y_{0}, z_{0}$ are the coordinates of the pristine state). The expansion along $x$, the pore direction $(<100>)$, is $13.1 \%=(552-488) / 488$. The expansion along both $y(<01-1>)$ and $z(<011\rangle)$ directions is $7.8 \%=(1315-1219) / 1219$. The apparent volume expansion of the particle is, therefore, calculated to be $=\left(x_{f}^{*} y_{f}^{*} z_{f}-x_{0}{ }^{*} y_{0}{ }^{*} z_{0}\right) /\left(x_{0}^{*} y_{0}^{*} z_{0}\right)=(1+13.1 \%)^{\star}(1+7.8 \%)^{\star}$ $(1+7.8 \%)=\sim 31.6 \%$. It is much lower than the $300 \%$ volume increase of conventional dense Si particles. From these in-situ
TEM observations, we conclude that the nanostructure of the MSS significantly reduces the apparent volume expansion and thus prevents lithiation-induced pulverization.

The in-situ TEM results and the excellent electrochemical performance demonstrated above indicate that MSS has a great potential to be used as a high capacity, highly stable anode material in the next-generation Li-ion batteries. This study identified the desired microstructures for stable porous $\mathrm{Si}$ anodes. In addition to electrochemical etched single crystalline Si waver, porous $\mathrm{Si}$ with the desired mesopore structure can also be obtained by several other scalable methods, including chemical etching of metallurgical grade and micron-sized silicon powder that is economically available in large quantities and suitable for large-scale production. The rational design of the novel structure developed in this work may also guide the development of other materials that may experience large volume changes during its operation processes.

\section{Methods}

Sample preparation. MSS samples were prepared by electrochemical etching of heavily boron-doped, $<100>$ oriented single crystal Si wafers $(<1 \mathrm{~m} \Omega \cdot \mathrm{cm}$ resistivity, Siltronix Inc.) by application of a constant current density of $\sim 225 \mathrm{~mA} \mathrm{~cm}^{-2}$ for $358 \mathrm{~s}$ in an aqueous $48 \%$ hydrofluoric acid (HF)/ethanol electrolyte (HF:EtOH $=3: 1, \mathrm{v}-\mathrm{v})^{34}$. A freestanding film of the MSS was then removed from the crystalline Si substrate by application of a constant current density of $\sim 10 \mathrm{~mA} \mathrm{~cm}^{-2}$ for $750 \mathrm{~s}$ in an aqueous HF/ethanol electrolyte $(\mathrm{HF}: \mathrm{EtOH}=1: 30, \mathrm{v}-\mathrm{v})$. The MSS film was washed several times with ethanol, then fractured by ultrasonication in ethanol to form $\sim 40-\mu \mathrm{m}$ particles, and dried for further use.

Carbon coating on the MSS particles was conducted using a vacuum chemical vapour deposition system. The MSS sample was loaded in a tube furnace, which then was evacuated at room temperature to a vacuum level of $\leq 1 \mathrm{mTorr}$. The furnace was heated to $600{ }^{\circ} \mathrm{C}$ at a rate of $10^{\circ} \mathrm{C} \mathrm{min}^{-1}$, and at $600^{\circ} \mathrm{C}$, the precursor gas (argon: acetylene $=9: 1$ ) was introduced. The furnace temperature was increased to $690^{\circ} \mathrm{C}$ and was maintained at that temperature for $30 \mathrm{~min}$. After carbon deposition, the furnace was cooled to room temperature in pure argon.

Structure characterization. XRD spectra were obtained on a Philips X'Pert X-ray diffractometer using a $\mathrm{Cu} \mathrm{K}_{\alpha}$ sealed tube $(\lambda=1.54178 \AA)$ at $0.5^{\circ} \mathrm{min}^{-1}$. SEM measurement was conducted on an FEI Helios Nanolab dual-beam focused ion beam SEM. After cycling, the samples were obtained from disassembled cells, washed three times with anhydrous dimethyl carbonate, and dried in the antechamber of the glove box under vacuum. Nitrogen sorption isotherms were obtained using a Quantachrome Autosorb automated gas sorption system at $-196^{\circ} \mathrm{C}$. Specific surface areas and pore size distributions were calculated using the Brunauer-Emmett-Teller theory and the Barrett-Joyner-Halenda method. Specific pore volumes were measured at a relative pressure of 0.99 .

In-situ TEM battery experiment. In-situ lithiation of the MSS particle was performed using a Nanofactory scanning tunnelling microscopy holder. Assembly of the in-situ TEM nano-cell battery was performed in an argon-filled glove box and transferred to the TEM in an argon-filled glove bag. The turbo pump in the TEM was pre-set to the maximum speed before loading the sample. Once the sample holder was inserted into the TEM, the sample environment was quickly pumped to vacuum. The total air exposure of the sample during loading into the TEM was $<2$ s. TEM measurements were performed using an FEI Titan operating at an accelerating voltage of $300 \mathrm{kV}$. During lithiation, a $-2 \mathrm{~V}$ potential was applied to the working electrode to drive the $\mathrm{Li}$ into the $\mathrm{Si}$ electrode through the $\mathrm{Li}_{2} \mathrm{O}$ solid electrolyte.

Electrochemical measurements. The MSS electrode was prepared by mixing an appropriate amount of the carbon-coated MSS sample with Super P (Timcal), and carboxymethyl cellulose sodium salt (Sigma Aldrich) in distilled water to form a slurry, which was then pasted on a copper foil (Pred. Materials International, Inc.). The Si loading in the mixture was kept at 46 and $40 \mathrm{wt} \%$. The binder of carboxymethyl cellulose sodium salt was kept at $20 \mathrm{wt} \%$ in all electrodes. After drying in vacuum at $70^{\circ} \mathrm{C}$, the electrodes were tested in coin cells with $\mathrm{Li}$ metal as the counter and the reference electrode. The electrolyte was $1 \mathrm{M} \mathrm{LiPF}_{6}$ in a mixture of ethylene carbonate and dimethyl carbonate in a 1:2 volume ratio with $10 \mathrm{wt} \%$ fluorinated ethylene carbonate as electrolyte additive. A microporous membrane (Celgard 3501) was used as the separator. The coin cells were assembled in an argon-filled glove box (MBraun Inc.). All the batteries were tested using galvanostatic charge-discharge protocols on a BT-2000 Arbin Battery Testing System (Arbin Instruments) at room temperature. For the electrodes with low Si loading $\left(\sim 0.5 \mathrm{mg} \mathrm{cm}^{-2}\right)$, tests were conducted within a voltage range of $0.05-1.5 \mathrm{~V}$. The 
batteries were cycled at a current density of $0.1 \mathrm{Ag}^{-1}$ for three cycles and then cycled at $1 \mathrm{Ag}^{-1}$ for long-term cycling stability or at different current densities for rate performance. The current density was calculated on the basis of the MSS weight. All of the specific weight-capacity values in this paper were calculated on the basis of the whole electrode weight including active material, binder and conductive carbon. For the electrodes with high Si loading $\left(\sim 2 \mathrm{mg} \mathrm{cm}^{-2}\right)$, tests were conducted within a voltage range of $0.005-1.0 \mathrm{~V}$. The batteries were cycled at a current density of $0.06 \mathrm{~mA} \mathrm{~cm}^{-2}$ for three cycles and then cycled at $0.75 \mathrm{~mA} \mathrm{~cm}^{-2}$ for charge (de-lithiation) and $0.5 \mathrm{~mA} \mathrm{~cm}^{-2}$ for discharge (lithiation). Cyclic voltammograms were measured on a CHI660d workstation $(\mathrm{CH}$ instruments, USA).

\section{References}

1. Armand, M. \& Tarascon, J. M. Building better batteries. Nature 451, 652-657 (2008).

2. Whittingham, M. S. Materials challenges facing electrical energy storage. MRS Bull. 33, 411-419 (2008).

3. Kasavajjula, U., Wang, C. S. \& Appleby, A. J. Nano- and bulk-silicon-based insertion anodes for lithium-ion secondary cells. J. Power Sources 163, 1003-1039 (2007)

4. Liu, X. H. et al. Size-dependent fracture of silicon nanoparticles during lithiation. ACS Nano 2, 1522-1531 (2012).

5. McDowell, M. T. et al. Studying the kinetics of crystalline silicon nanoparticle lithiation with in situ transmission electron microscopy. Adv. Mater. 24, 6034-6041 (2012).

6. Gu, M. et al. In situ TEM study of lithiation behavior of silicon nanoparticles attached to and embedded in a carbon matrix. ACS Nano 6, 8439-8447 (2012).

7. Kim, H. J., Seo, M., Park, M.-H. \& Cho, J. A critical size of silicon nano-anodes for lithium rechargeable batteries. Angew. Chem. Int. Ed. 49, 2146-2149 (2010).

8. Kovalenko, I. et al. A major constituent of brown algae for use in high-capacity Li-ion batteries. Science 334, 75-79 (2011).

9. Liu, G. et al. Polymers with tailored electronic structure for high capacity lithium battery electrodes. Adv. Mater. 23, 4679-4683 (2011).

10. Chan, C. K. et al. High-performance lithium battery anodes using silicon nanowires. Nat. Nanotech. 3, 31-35 (2008).

11. Wu, H. et al. Stable cycling of double-walled silicon nanotube battery anodes through solid-electrolyte interphase control. Nat. Nanotech. 7, 310-315 (2012).

12. Yao, Y. et al. Interconnected silicon hollow nanospheres for lithium-ion battery anodes with long cycle life. Nano Lett. 11, 2949-2954 (2011).

13. Wu, H. et al. Engineering empty space between Si nanoparticles for lithium-ion battery anodes. Nano Lett. 12, 904-906 (2012).

14. Magasinki, A. et al. High-performance lithium-ion anodes using a hierarchical bottom-up approach. Nat. Mater. 9, 353-358 (2010).

15. Li, X. L. et al. Hollow core-shell structured porous $\mathrm{Si}-\mathrm{C}$ nanocomposites for Li-ion battery anodes. J. Mater. Chem. 22, 11014-11017 (2012).

16. Chen, X. L. et al. Conductive rigid skeleton supported silicon as highperformance Li-ion battery anodes. Nano Lett. 12, 4124-4130 (2012).

17. Park, M. H. et al. Silicon nanotube battery anodes. Nano Lett. 9, 3844-3847 (2009).

18. Song, T. et al. Arrays of sealed silicon nanotubes as anodes for lithium ion batteries. Nano Lett. 10, 1710-1716 (2010).

19. Evanoff, K., Magasinski, A., Yang, J. B. \& Yushin, G. Nanosilicon-coated graphene granules as anodes for Li-ion batteries. Adv. Energy Mater. 1, 495-498 (2011).

20. Ge, M. Y., Rong, J. P., Fang, X. \& Zhou, C. W. Porous doped silicon nanowires for lithium ion battery anode with long cycle life. Nano Lett. 12, 2318-2323 (2012).

21. Hu, Y. S. et al. Superior storage performance of a Si@SiOx/C nanocomposite as anode material for lithium-ion batteries. Angew. Chem. Int. Ed. 47, 1645-1649 (2008).

22. Hwang, T. H., Lee, Y. M., Kong, B. S., Seo, J. S. \& Choi, J. W. Electrospun coreshell fibers for robust silicon nanoparticle-based lithium ion battery anodes. Nano Lett. 12, 802-807 (2012).

23. Zhao, X., Hayner, C. M., Kung, M. C. \& Kung, H. H. In-plane vacancy-enabled high-power Si-graphene composite electrode for lithium-ion batteries. Adv. Energy Mater. 1, 1079-1084 (2011).

24. Kim, H. J., Han, B. H., Choo, J. B. \& Cho, J. Three-dimensional porous silicon particles for use in high-performance lithium secondary batteries. Angew. Chem. Int. Ed. 47, 10151-10154 (2008).
25. Bang, B. M., Lee, J. I., Kim, H. J., Cho, J. \& Park, S. J. High-performance macroporous bulk silicon anodes synthesized by template-free chemical etching. Adv. Energy Mater. 2, 878-883 (2012).

26. Chen, D. Y. et al. Reversible lithium-ion storage in silver-treated nanoscale hollow porous silicon particles. Angew. Chem. Int. Ed. 51, 2409-2413 (2012).

27. Gowda, S. R. et al. Three-dimensionally engineered porous silicon electrodes for Li ion batteries. Nano Lett. 12, 6060-6065 (2012).

28. Jia, H. P. et al. Novel three-dimensional mesoporous silicon for high power lithium-ion battery anode material. Adv. Energy Mater. 1, 1036-1039 (2011).

29. Thakur, M., Sinsabaugh, S. L., Isaacson, M. J., Wong, M. S. \& Biswal, S. L. Inexpensive method for producing macroporous silicon particulates (MPSPs) with pyrolyzed polyacrylonitrile for lithium ion batteries. Sci. Rep. 2, 795 (2012).

30. Yi, R., Dai, F., Gordin, M. L., Chen, S. R. \& Wang, D. H. Micro-sized Si-C composite with interconnected nanoscale building blocks as high-performance anodes for practical applicaiton in lithium-ion batteries. Adv. Energy Mater. 3, 295-300 (2013).

31. Zhang, H. G. \& Braun, P. V. Three-dimensional metal scaffold supported bicontinuous silicon battery anodes. Nano Lett. 12, 2778-2783 (2012).

32. Wu, H. et al. Stable Li-ion battery anodes by in-situ polymerization of conducting hydrogel to conformally coat silicon nanoparticles. Nat. Commun. 4, 1943 (2013).

33. Liu, N. et al. A pomegranate-inspired nanoscale design for large-volumechange lithium battery anodes. Nat. Nanotech. 9, 187-192 (2014).

34. Uhlir, A. Electrolytic shaping of germanium and silicon. Bell Syst. Tech. J. 35, 333-347 (1956).

35. Sailor, M. J. Porous Silicon in Practice: Preparation, Characterization and Applications (Wiley-VCH, 2012).

36. Park, J. H. et al. Biodegradable luminescent porous silicon nanoparticles for in vivo applications. Nat. Mater. 8, 331-336 (2009).

37. $\mathrm{Gu}$, L. et al. In vivo time-gated fluorescence imaging with biodegradable luminescent porous silicon nanoparticles. Nat. Commun. 4, 2326 (2013).

38. Salonen, J., Laine, E. \& Niinisto, L. Thermal carbonization of porous silicon surface by acetylene. J. Appl. Phys. 91, 456-461 (2002).

39. Lee, S. W., McDowell, M. T., Choi, J. W. \& Cui, Y. Anomalous shape changes of silicon nanopillars by electrochemical lithiation. Nano Lett. 11, 3034-3039 (2011).

40. Yang, H. et al. Orientation-dependent interfacial mobility governs the anisotropic swelling in lithiated silicon nanowires. Nano Lett. 12, 1953-1958 (2012).

\section{Acknowledgements}

This work was supported by the Assistant Secretary for Energy Efficiency and Renewable Energy, Office of Vehicle Technologies, of the US Department of Energy (DOE) under Contract No. DE-AC02-05CH11231, Subcontract No. 18769, under the Batteries for Advanced Transportation Technologies (BATT) program, and by the US National Science Foundation under Grant No. DMR-1210417. A portion of the research was performed at the William R. Wiley Environmental Molecular Sciences, a national scientific user facility sponsored by the DOE's Office of Biological and Environmental Research and located at Pacific Northwest National Laboratory.

\section{Author contributions}

X.L., M.J.S., J.-G.Z. and J.L. conceived and designed the research. X.L., M.G., S.H., R.K. and X.C. performed the experiments and analyzed the data. X.L., M.G., S.H., C.W., M.J.S., J.-G.Z. and J.L. wrote the manuscript.

\section{Additional information}

Supplementary Information accompanies this paper at http://www.nature.com/ naturecommunications

Competing financial interests: The authors declare no competing financial interests.

Reprints and permission information is available online at http://npg.nature.com/ reprintsandpermissions/

How to cite this article: $\mathrm{Li}, \mathrm{X}$. et al. Mesoporous silicon sponge as an anti-pulverization structure for high-performance lithium-ion battery anodes. Nat. Commun. 5:4105 doi: 10.1038/ncomms5105 (2014). 